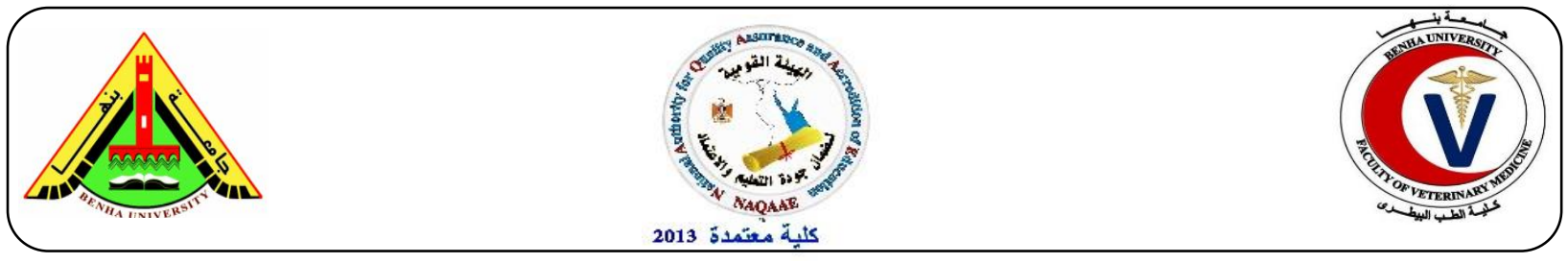

\title{
Improving performance of Pneumo-4 vaccine using montanide oil
}

\author{
Gabr F. El-Bagoury ${ }^{1}$, Ayman S. El-Habbaa ${ }^{1}$, Effat L. El-Sayed ${ }^{2}$, Manal Abd El-Aziz ${ }^{2}$ \\ ${ }^{1}$ Department of Virology, Faculty of Veterinary Medicine, Benha University. ${ }^{2}$ Rinderpest-Like Disease \\ Department, Veterinary Serum and Vaccine Research Institute, Abbasia, Cairo.
}

\begin{abstract}
A B S T R A C T
Selecting of the adjuvant is one of the keys for the success of the vaccine application. Oil adjuvants, water-in-oil-in-water $(\mathrm{W} / \mathrm{O} / \mathrm{W})$, have proved their efficacy in bovine vaccine as they induce rapid and long term immune response with various antigens. The present study was aimed towards improving and upgrading the locally prepared inactivated combined vaccine (Pneumo-4) containing Infectious Bovine Rhinotracheitis (IBR), Bovine Viral Diarrhoea (BVD), Parainfleunza type 3 (PI-3) and Bovine Respiratory syncytial (BRS) viruses using Montanide oil ISA206 as adjuvant and compare its efficacy with the commercial gel adjuvanted one. Both the commercial and prepared oil Pneumo-4 vaccines were administered twice to 6-9 months old seronegative calves through intramuscular (I/M) route with dose $5 \mathrm{ml}, 21$ days apart. Humoral immune response was followed up in the sera of vaccinated calves using serum neutralization test (SNT). The result revealed that both adjuvanted vaccines gave a significant effect on all vaccinated animals but the oil adjuvanted vaccine was particularly better and effective than gel adjuvanted where oil adjuvanted vaccine induced early intense immune response and showed prolongation of antibody secretion in comparison to the Aluminium Hydroxide gel.
\end{abstract}

Keywords: Pneumo-4 Vaccine, ISA 206, SNT.

(http://www.bvmj.bu.edu.eg)

(BVMJ, 33 (2) : 249-258, DECEMBER, 2017)

\section{INTRODUCTION}

Bovine respiratory disease (BRD) is a worldwide health concern in stocker, background and feedlot cattle causing morbidity and mortality in young cattle with major economic losses to the producer (Windeyeo and Leslie, 2012 and Hilton, 2014).

$\mathrm{BRD}$ is a respiratory disease complex that occurs most often within 4 weeks after weaning. The weaning process is a stressful time for calves. At that time, calves are handled, commingled, and shipped to other locations. During this period, calves may be exposed to many infectious agents that cause
BRD (Taylor et al., 2010). BRD is a "multifactorial syndrome" that is dependent on a number of different causes. A viral infection combines with a bacterial agent, which is often then aggravated by stress (Kasimanickam, 2010).

The most common viruses involved with BRD include Bovine Viral Diarrhea (BVD), Infectious Bovine Rhinotracheitis (IBR), Bovine Respiratory Syncytial (BRS), and Parainfluenza Type-3 (PI-3V) Viruses. Exposure to these viruses can cause severe damage to the respiratory tract of calves creating opportunities for bacteria to then settle in the lungs (Taylor et al., 2010; Kasimanickam, 2010; Currin and Dee Whittier, 2009). 
Fortunately, many of these agents can be prevented through vaccination programs. Selecting the adjuvant is one of the keys for the success of the vaccine. There are two main technologies for veterinary adjuvant in the field can fit with multiple expectation as safety and efficacy in various species such as aluminum salts and emulsion (Lindbald, 2004 and Oda et al., 2006).

The present study aims for preparation and evaluation of combined inactivated oily adjuvant vaccine using ISA 206 and compare the efficacy of this prepared oil vaccine with the gel adjuvant one.

\section{MATERIAL AND METHODS}

\subsection{Viral Strains:}

\subsubsection{Bovine Viral Diarrhea (BVD) virus:}

Egyptian Bovine Viral Diarrhea virus (BVDV), (Iman Strain) with a titer of $7 \log _{10}$ TCID $_{50} / \mathrm{ml}$ first isolated and identified by Baz, (1975).

\subsubsection{Infectious Bovine Rhino-tracheitis (IBR) virus:}

A reference Egyptian strain of Infectious Bovine Rhino-tracheitis virus (IBRV), (Abou Hammad Strain) with a titer of $8 \log _{10} \mathrm{TCID}_{50} / \mathrm{ml}$. It was firstly isolated by Hafez et al., (1976).

\subsubsection{Para Influenza-3 (PI-3) virus:}

A reference Egyptian strain of Para. Influenza-3 virus (Strain 45) with a titer of $8 \log _{10} \mathrm{TCID}_{50} / \mathrm{ml}$, which was isolated and identified by Singh and Baz, (1966).

\subsubsection{Bovine Respiratory Syncytial (BRS)} virus:

Reference strain "375L" of BRS virus with a titer $6 \log _{10} \mathrm{TCID}_{50} / \mathrm{ml}$.
All virus entities were adapted, propagated and titrated on Madin Darby Bovine Kidney (MDBK) cell culture, which has been proved free of any adventitious agents; these vaccinal viral strains were gently supplied by Rinder Pest-like Disease Research Dept. Vet. Serum and Vaccine Research Institute (VSVRI), Cairo. The viral strains were stored at $-70{ }^{\circ} \mathrm{C}$ and were used as seed virus for preparation of the vaccine and in Serum Neutralization Test (SNT).

\subsection{Madin-Darby Bovine Kidney $(M D B K)$ cell line:}

It was supplied by the Animal Research Institute, Pirbright, UK. It was propagated at Rinder Pest-like Disease Research Dept., VSVRI, Abbasia, Cairo, Egypt by using of minimum Essential Medium (MEM) with Eagle's salts and with $10 \%$ new born calf serum (Wellenberg, et al., 2002). These cells were used for virus propagation and titration for viruses and for SNT.

\subsection{Commercial inactivated Pneumo-4 vaccine:}

Pneumo-4 vaccine adjuvant with aluminum hydroxide gel, containing tissue culture adapted BVDV, IBRV, PI-3V and BRSV after being inactivated using Binary Ethylenimine (BEI). It was obtained from department of Rinderpest-like diseases, VSVRI, Abbasia, Cairo, Egypt. It was used for vaccination of animals by intramuscular inoculation in two doses of $5 \mathrm{ml}, 3$ weeks apart.

\subsection{Formulation of Pneumo-4 Vaccine using montanide oil ISA 206 adjuvant:}

All reference viruses (BVD, IBR, PI3 and BRS viruses) were propagated separately in MDBK. Then the viruses were harvested and titrated on MDBK cell line. BVDV, IBRV, PI-3V and BRSV have the titers of $7 \quad \log 10 \quad$ TCID50/ml, $8 \quad \log 10$ 
TCID50/ml, $8 \log 10$ TCID50/ml and $6 \log 10$ TCID50/ml, respectively; then inactivation process was initiated with a final concentration of $0.01 \% \mathrm{M}$ BEI (Aldrich chemical Co. Ltd., Grilling ham Dorset, England) at $37^{\circ} \mathrm{C}$ and stopped by addition of cold Sodium Thiosulphate (Loba Chemine Co. Ltd., Mumbai, India) with a final concentration of $2 \%$ (El-Sabbagh et al., 1995 and Samira et al., 2001). The inactivated virus suspensions were combined as equivalent volumes then this aqueous phase was mixed weight by weight $(\mathrm{W} / \mathrm{W})$ with Montanide ISA 206 oil adjuvant (SEPPIC, France) to make water-in-oil-inwater suspension. The prepared vaccine was dispensed into specific bottles and stored at $4^{\circ} \mathrm{C}$ until used for checking sterility, safety, and evaluation of the vaccine.

\subsection{Quality control of the prepared vaccine:}

Sterility and safety was evaluated for the prepared Pneumo-4 vaccine (CFR, Code of Federal Regulation of USA, 1986). Sterility test was applied to confirm that the prepared vaccine was free from bacterial and fungal contamination using nutrient agar, thioglycolate broth (for bacterial detection) and Sabouraud's dextrose agar (for fungal detection). Safety of the inactivated viruses before addition of adjuvant was applied on tissue culture and the safety of the prepared oil adjuvant Pneumo-4 vaccine after adjuvant was applied in White Swiss mice and calves by injection of $0.2 \mathrm{ml}$ intraperitoneally (I/P) and $50 \mathrm{ml}$ (10 vaccinal dose) intramuscularly (I/M) of the vaccine, respectively.

\subsection{Animal and Experimental design:}

\subsubsection{Animals:}

\subsubsection{White Swiss mice:}

Ten white Swiss mice of 10-15 gm weight used for safety test of the prepared vaccine obtained from
Laboratory Animals Department, VSVRI, Abbasia, Cairo.

\subsubsection{Calves:}

Fifteen apparently healthy calves aged 6-9 months were sero-negative for all viral components of the prepared vaccine as screened by SNT. Two calves were used for safety of the prepared vaccine and the other thirteen calves were used for potency test, divided into three groups:

Group (I) consists of 5 calves vaccinated with prepared oil adjuvanted vaccine.

Group (II) consists of 5 calves vaccinated with commercial vaccine.

In both groups (I \& II), each calf was vaccinated by two doses, 21 days apart, each dose $5 \mathrm{ml} \mathrm{I} / \mathrm{M}$.

Group (III) containing 3 calves was used as non-vaccinated control. Each calf injected with the same dose and root with physiological saline.

\subsubsection{Pregnant cows:}

Three apparently healthy pregnant cows were used for evaluation of the prepared inactivated combined vaccine and after delivery the newly born were naturally fed and get under the same condition. The pregnant cows were vaccinated two months before inoculation and boostered 21 days later.

\subsection{Sampling:}

Serum samples were collected from all calves of different tested groups just before 1st vaccination, and then weekly postvaccination until the first month and then monthly until the end of the experiment. All collected sera were inactivated at $56{ }^{\circ} \mathrm{C}$ for $30 \mathrm{~min}$. in water bath for inactivation of nonspecific antiviral substances then stored at $20^{\circ} \mathrm{C}$ till used in serological test. All serum samples were serologically assayed for 
detection immune response and duration of immunity.

\subsection{Serum Neutralization Test (SNT):}

All serum samples were tested using serum neutralization test for detection of specific neutralizing antibodies against all vaccinal viruses of Pneumo-4 commercial and prepared oil adjuvants. SNT applied in micro-titration plate technique following the procedure described by Rossi and Kiessel, (1971). The Serum Neutralizing Antibody titers of the tested serum samples were expressed as $\log 10$ of the reciprocal serum dilution that protects $>50 \%$ of micro titration plate dilution wells, and these following the calculation formula of Reed and Muench, (1938).

\section{RESULTS}

\subsection{Sterility and safety of the vaccine:}

The prepared ISA 206 oil adjuvanted Pneumo-4 vaccine was free from aerobic and anaerobic bacteria and fungi. It was also safe and gave satisfactory results indicated by absence of cytopathic effect on tissue culture, and absence of deaths in inoculated mice and absence of local and systemic reactions with no rise in body temperature.

\subsection{Humeral immune response of vaccinated} calves using SNT:

The mean neutralizing antibody in sera of calves vaccinated with oil adjuvant Pneumo-4 vaccine (Group I) were gradually increased from 14th day post vaccination (DPV) within the minimal protective level for all viruses then reached highest level at 2th month post vaccination (MPV) and still within the minimal acceptable titer of protective level till the end of the experiment (9 months) for all viral components of the prepared vaccine (IBR, BVD, PI-3 and BRS virus), (Table 1 and Figure 1).

For calves vaccinated with aluminum hydroxide gel adjuvant Pneumo-4 vaccine (Group II); the mean neutralizing antibody in their sera were gradually increased from 14th DPV but were lower than the minimal acceptable titer (Fulton et al., 1995) then the minimal protective level for all viruses from 21th DPV and persisted within the minimal acceptable titer of protective level till $6 \mathrm{MPV}$ for all viral components of the prepared vaccine (IBR, BVD, PI-3 and BRS virus), (Table 2 and Figure 2).

\subsection{Humeral immune response of vaccinated pregnant cows and their newly born calves using SNT:}

The mean neutralizing antibody in sera of pregnant cows vaccinated with oil adjuvant Pneumo-4 vaccine (Group III) were increased to reach the protective levels for all viral components of the vaccine at 21th DPV and persisted at minimal acceptable titer of protective level till time of parturition. For calves delivered from these vaccinated cows, the mean neutralizing antibody in their sera were within the minimal protective level from the first week post parturition (WPP) and persisted within the protective level till 8 WPP for all viral components of the prepared vaccine (IBR, BVD, PI-3 and BRS virus), (Table 3). 
Table (1) Mean neutralizing antibody titer against IBRV, BVDV, PI-3V and BRSV in sera of calves vaccinated with Pneumo-4 vaccine adjuvanted with Montanide oil (ISA206).

\begin{tabular}{ccccccccccccc}
\hline Vaccinated & \multicolumn{10}{c}{ Neutralizing antibody titers expressed in $\log _{10}$} \\
animal & $0 \mathrm{~d}^{*}$ & $14 \mathrm{~d}$ & $21 \mathrm{~d}^{* *}$ & $1 \mathrm{M}$ & $2 \mathrm{M}$ & $3 \mathrm{M}$ & $4 \mathrm{M}$ & $5 \mathrm{M}$ & $6 \mathrm{M}$ & $7 \mathrm{M}$ & $8 \mathrm{M}$ & $9 \mathrm{M}$ \\
\hline IBR virus & 0.34 & 1.2 & 1.56 & 1.62 & 2.46 & 2.4 & 2.56 & 1.98 & 1.68 & 1.08 & 1.08 & 0.98 \\
BVD virus & 0.3 & 0.84 & 1.4 & 1.62 & 2.1 & 2.16 & 1.8 & 1.44 & 1.32 & 1.08 & 0.99 & 0.84 \\
PI-3 virus & 0.45 & 1.08 & 1.44 & 1.74 & 2.4 & 2.52 & 2.22 & 1.98 & 1.68 & 1.38 & 1.26 & 1.08 \\
BRS virus & 0.45 & 0.9 & 1.38 & 1.74 & 2.34 & 2.1 & 1.8 & 1.62 & 1.44 & 1.14 & 1.11 & 0.75
\end{tabular}

Control group

Negative immune response, the titer ranged between $0.15-0.45$

$* 1$ st dose, $* *$ 2nd dose.

Minimum acceptable titer of protective level expressed in $\log _{10}$ against, IBRV $=0.6$,

$\mathrm{BVDV}=0.9, \mathrm{PIV}-3=0.6, \mathrm{BRSV}=0.6$ (Fulton et al., 1995).

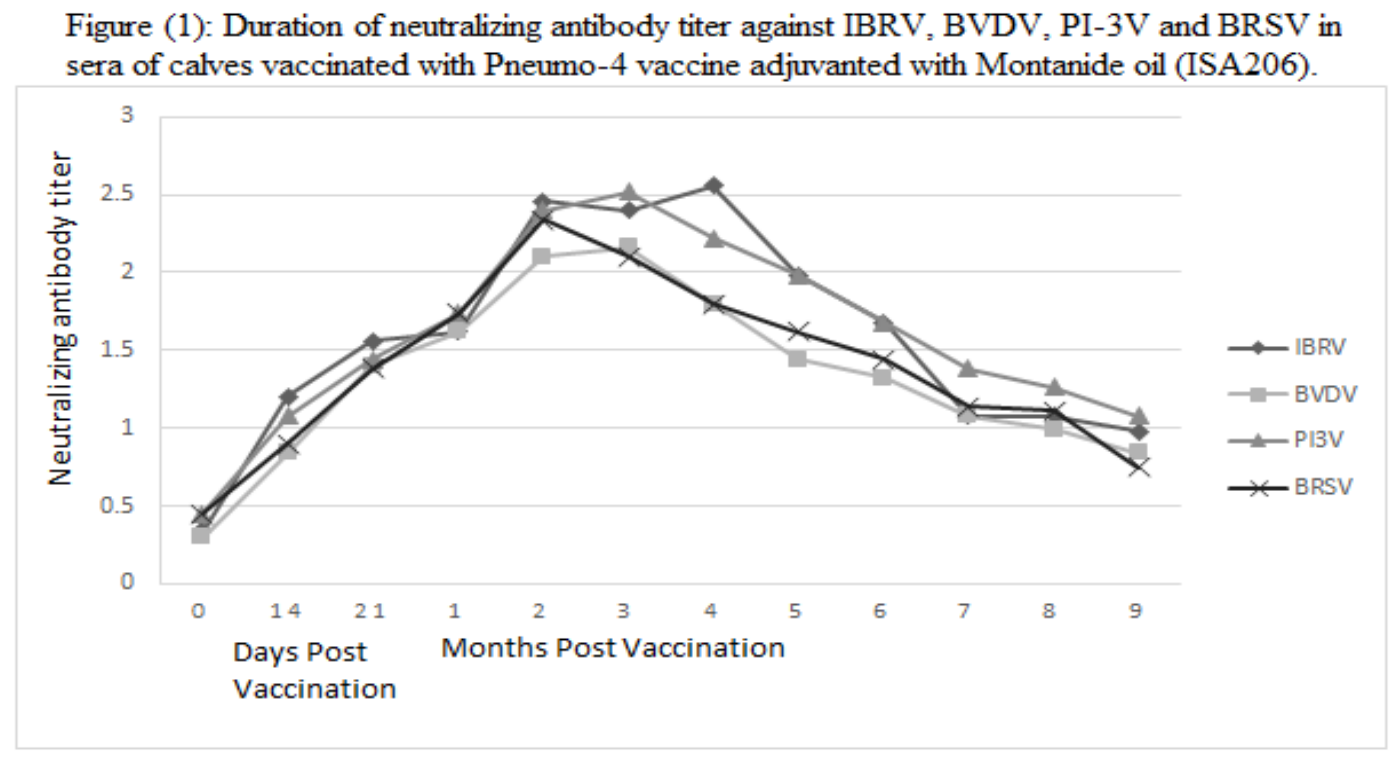

Table (2) Mean neutralizing antibody titer against IBR, BVD, PI-3 and BRS viruses in sera of calves vaccinated with pneumo-4 vaccine adjuvanted with Aluminum hydroxide gel:

\begin{tabular}{|c|c|c|c|c|c|c|c|c|c|c|c|c|}
\hline \multirow{2}{*}{$\begin{array}{c}\text { Vaccinated } \\
\text { animal }\end{array}$} & \multicolumn{12}{|c|}{ Neutralizing antibody titers expressed in $\log _{10}$} \\
\hline & $0 \mathrm{~d}^{*}$ & $14 \mathrm{~d}$ & $21 d^{* *}$ & $1 \mathrm{M}$ & $2 \mathrm{M}$ & $3 \mathrm{M}$ & $4 \mathrm{M}$ & $5 \mathrm{M}$ & $6 \mathrm{M}$ & $7 \mathrm{M}$ & $8 \mathrm{M}$ & $9 \mathrm{M}$ \\
\hline IBR virus & 0.24 & 0.48 & 0.69 & 1.17 & 1.65 & 1.53 & 1.29 & 1.05 & 0.75 & 0.51 & 0.37 & 0.24 \\
\hline BVD virus & 0.21 & 0.51 & 0.78 & 1.35 & 1.71 & 1.59 & 1.32 & 1.11 & 0.93 & 0.69 & 0.51 & 0.3 \\
\hline PI-3 virus & 0.24 & 0.63 & 0.81 & 1.2 & 1.71 & 1.62 & 1.38 & 1.32 & 1.05 & 0.81 & 0.57 & 0.33 \\
\hline BRS virus & 0.21 & 0.54 & 0.93 & 1.38 & 1.62 & 1.56 & 1.29 & 1.05 & 0.78 & 0.57 & 0.39 & 0.18 \\
\hline $\begin{array}{l}\text { Control } \\
\text { group }\end{array}$ & \multicolumn{12}{|c|}{ Negative immune response, the titer ranged between $0.15-0.45$} \\
\hline
\end{tabular}


Figure (2): Duration of neutralizing antibody titer against IBR, BVD, PI-3 and BRS viruses in sera of calves vaccinated with pneumo-4 commercial vaccine adjuvanted with Aluminum hydroxide gel.

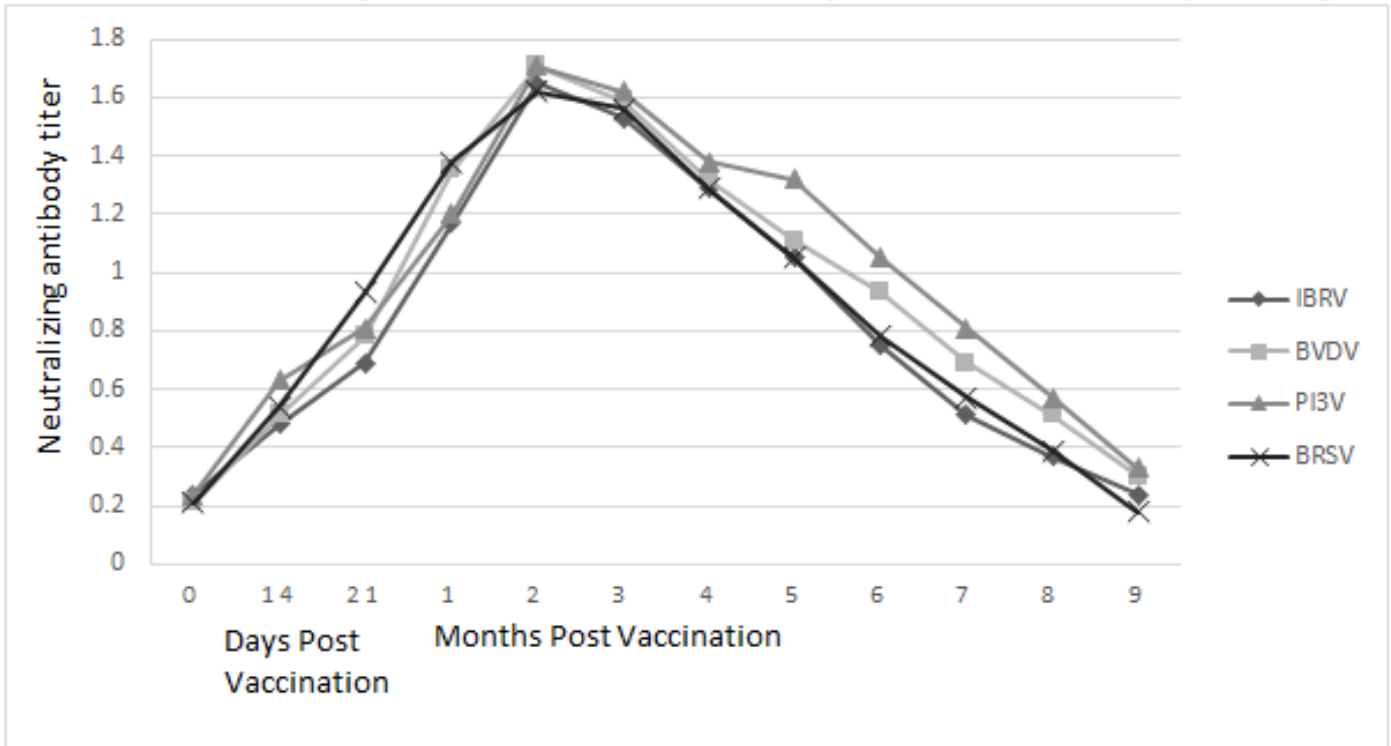

Table (3) Mean serum neutralizing antibody titer against IBR, BVD, PI-3 and BRS viruses in sera of vaccinated pregnant dams with Penmuo-4 oil adjuvant and their offspring:

\begin{tabular}{|c|c|c|c|c|c|c|c|c|c|}
\hline \multirow{2}{*}{$\begin{array}{c}\text { Type of } \\
\text { Virus }\end{array}$} & \multicolumn{9}{|c|}{ Neutralizing antibody titers expressed in $\log _{10}$} \\
\hline & \multicolumn{3}{|c|}{ Sera of dams at } & \multicolumn{6}{|c|}{ Sera of offspring at weeks post parturition } \\
\hline IBR virus & 0.6 & 1.2 & 2.1 & 1.9 & 1.6 & 1.35 & 1.15 & 1 & 0.8 \\
\hline BVD virus & 0.3 & 0.9 & 1.7 & 1.6 & 1.6 & 1.4 & 1.15 & 0.95 & 0.7 \\
\hline PI-3 virus & 0.4 & 1 & 1.9 & 1.7 & 1.5 & 1.25 & 1.1 & 0.8 & 0.6 \\
\hline BRS virus & 0.3 & 0.9 & 1.7 & 1.6 & 1.35 & 1.15 & 0.95 & 0.7 & 0.45 \\
\hline
\end{tabular}




\section{DISCUSSION}

Bovine Respiratory Disease (BRD) is the most common disease problem encountered in stocker or feedlot calves causing economic losses.

The use of inactivated vaccines produces good result for protection of calves against BRD. The efficacy of these vaccines is variable depending on many factors. Adjuvants which considered one of the most important factors in vaccine formulation, so the selection of vaccine adjuvant must be done according to several criteria with the goal to obtain a good balance between safety and immunogenicity. Most commonly used adjuvants in bovine vaccine are oil adjuvant and aluminum hydroxide gel, the later generally enhance humoral immune response but several booster vaccinations are necessary to maintain a long term immune response. While oil emulsion such as $(\mathrm{W} / \mathrm{O} / \mathrm{W})$ have proved their efficacy in bovine vaccine as they can induce rapid and long term immune response. So this study aimed to prepare experimental batch of inactivated polyvalent vaccine containing IBR, BVD, PI-3 and BRS viruses, adjuvanted with Montanid oil (ISA 206) and compare its efficacy with commercial gel adjuvanted one.

This trial gave satisfactory results concerning the laboratory evaluation of the prepared combined inactivated oil adjuvant for sterility, safety and potency testing. Sterility test of the prepared oil vaccine showed complete absence of any contamination on the inoculated media for 2 weeks post inoculation. Also, the results of safety test showed a good safety profile for Montanide oil adjuvant even in calves inoculated with 10 times of vaccinal dose showed neither local nor systemic post vaccinal reaction and no development of any clinical signs or any abnormalities till the end of the experiment.

Regarding potency evaluation of the prepared oil adjuvant and commercial vaccines, where immune stimulation due to the vaccine was measured by the neutralization test, the results obtained from Table (1) and Figure (1) revealed that all vaccinated calves in Group (I) developed neutralizing antibodies against all viral components of the vaccine showing clearly that Montanide oil prepared vaccine is highly immunogenic and inducing of humoral immune response more than 9 MPV. Concerning Group (II) of calves vaccinated with commercial Pneumo-4 vaccine, the result revealed that the duration of immunity lasted up to 6 MPV (Table 2 and Figure 2). This agrees with results of Ellis et al., (1995) Lindbald, (2004); De Gregorio et al., (2008) and El-Bagoury et al., (2012) whom reported that duration of immunity elicited by aluminium Hydroxide gel vaccine was short lived and antibody concentration rapidly falls over periods of 4-6 MPV.

The result represented in Table (3) showed that vaccinated dams developed high titer of neutralizing antibody at pre and post parturition. Also, maternal immunity was measured in sera of offspring from vaccinated cows for $12 \mathrm{WPP}$, proved high correlation with the titer of dams, which indicates high potency of the prepared vaccine with adequate neutralizing antibody titer within the minimal protective level. This agreed with the study which reported that the minimum accepted neutralizing antibody titer to be $0.9 \log _{10}$ for BVD virus, and $0.6 \log _{10}$ for IBR, PI-3 and BRS viruses (Fulton et al., 1995).

In conclusion, the prepared combined inactivated vaccine adjuvanted with the Montanide oil (ISA206) has been proved sterile, entirely safe and greatly effective 
giving duration of immunity more than 9 M.P.V. compared to that of the commercial vaccine adjuvanted with Aluminum Hydroxide gel which gives a duration of up to $6 \mathrm{MPV}$.

\section{REFERENCES}

Baz, T.I. 1975. Isolation, characterization and serological studies on BVD-MD virus in Egypt. Ph.D. Thesis. Fac. Vet. Med. Cairo Univ.

CFR, Code of Federal Regulation of USA 1986. Published by the office of the federal register national archives and Record administration. Animal and animal products 9\1986.

Currin, J.; Dee Whittier, W. 2009. Recognition and Treatment of Bovine Respiratory Disease Complex. Virginia Cooperative Extension -Maryland Regional College of Veterinary.

De Gregorio, E.; Tritto, E.; Rappuoli, R. 2008. Alum. Adjuvanticity: unraveling a century old mystery. Eur. J. Immunol. 38: 2068-2071.

El-Bagoury, G.F.; El-Nahas, E.M.; Abd ElFadil, M.R.; Ghaley, H.M. 2012. Evaluation of maternal antibody in calves born from cow dam vaccinated with inactivate Pneumo-5 vaccine. BVMJ, 23(2):9-15.

Ellis, J.A.; Hassard, L.E.; Morely, P.S. 1995. Bovine Respiratory Syncytial virus specific immune response in calves after inoculation with commercially available vaccine. J. Am. Vet. Med. Assoc. 206:354-361.

El-Sabbagh, M.M.; Samira, S.T.; Ghally, H.M.; Saad, M.S. 1995. Binary Ethylene Imine as an inactivant for Infectious Bovine Rhino-tracheitis (IBR), Bovine Viral Diarrhea (BVD), and Para-Influenza-3 (PI-3) viruses and it's application for vaccine production. Beni-Suef, Vet. Med. Res. 5(2): 29-57.
Fulton, R.W.; Confer, A.W.; Burge, L.J.; Perino, L.J.; d'Offay, J.M.; Payton, M.E.; Mock, R.E. 1995. Antibodies responses by cattle after vaccination with commercial viral vaccines containing BHV-1, BVDV, PIV-3, BRSV immunogens and subsequent revaccination at day 140 . Vaccine, 13:725-733.

Hafez, S.M.; Thanaa, I. Baz; Mohsen, A.Y.; Monira, H. 1976. Infectious Bovine Rhino-tracheitis in Egypt. Isolation and serological identification of the virus. J. Egypt, Vet. Med. Assoc. 36(1): 129139.

Hilton, W.M. 2014. BRD in 2014. Where have we been, where we are now, and where do we want to go. Anim. Healt Res. Rev. 15(2): 120-2.

Lindbald, E.B. 2004. Aluminum adjuvants in retrospect and prospect - vaccine. Sep 9, 22(27-28): 3658-68.

Oda, K.; Tsukahara, F.; Kutoba, S.; Kida, K.; Kitajima, T.; Hashimoto, S. 2006. Emulsifier content and side effect of oil based adjuvant in swine - Research in Veterinary science. 81, 51-57.

Kasimanickam, R. 2010. Bovine Respiratory Disease "Shipping Fever" in Cattle, Washington State University Veterinary Medicine Extension.

Reed, L.T.; Muench, H.A. 1938. Simple method of calculating fifty percent end point. Am. J. Hyg. 27: 493-8.

Rossi, C.R.; Kiessel, G.K. 1971. Microtitre tests for detecting antibody in bovine serum Para-Influenza-3 virus (PI-3), Infectious Bovine Rhinotracheitis virus (IBR) and Bovine Viral Diarrhea (BVD) Microbiolo. 22:32-26.

Samira, S.T.; El-Sabbagh, M.M.; Ghally, H.M. 2001. Preparation of combined inactivated BVD, IBR, PI-3 and Respiratory Syncytia Virus (BRSV). 
Egypt, Vet. Med. Assoc., 61(4): 251:263.

Singh, K.V.; Baz, Thanaa, I. 1966. Isolation of Para-Influenza -3 virus from water buffaloes in Egypt. Nature land, 210: 616-625.

Taylor, J.D.; Fulton, R.W.; Lehenbauer, T.W.; Step, D.L.; Confer, A.W. 2010. "The epidemiology of bovine respiratory disease: What is the evidence for predisposing factors". The Canadian veterinary journal, La revue veterinaire canadienne, 51 (10): 1095102.
Wellenberg, G.J., Verstraten, E.R.A.M., Jongejan F., Van Oirschot, J.T. 2002. Susceptibility of bovine umbilical cord endothelial cells to bovine herpesviruses and pseudocowpox virus. Vet. Res. Comm., 26(5): 407417.

Windeyeo M.C.; Leslie, K.E. 2012. The effects of viral vaccination of dairy heifer calves on the incidence of respiratory diseases, mortality and growth. Vol. 95, issue 11, pages 67406749. 